\title{
CORRECTION
}

\section{Correction to: The consensus number of a cryptocurrency}

\author{
Rachid Guerraoui ${ }^{1} \cdot$ Petr Kuznetsov ${ }^{2} \cdot$ Matteo Monti $^{1} \cdot$ Matej Pavlovic $^{1}$ (i) $\cdot$ Dragos-Adrian Seredinschi $^{1}$
}

Accepted: 11 February 2022 / Published online: 23 February 2022

c) Springer-Verlag GmbH Germany, part of Springer Nature 2022

\section{Correction to: Distributed Computing} https://doi.org/10.1007/s00446-021-00399-2

In the original publication of the article, the title of the article was incorrectly published as "The consensus number of a cryptocurrency (extended version)". The corrected version is "The consensus number of a cryptocurrency". This erratum corrects the same.
The original article has been updated.

Publisher's Note Springer Nature remains neutral with regard to jurisdictional claims in published maps and institutional affiliations.

The original article can be found online at https://doi.org/10.1007/s00446-021-00399-2.

$\bowtie$ Matej Pavlovic

matopavlovic@gmail.com

Rachid Guerraoui

rachid.guerraoui@epfl.ch

Petr Kuznetsov

petr.kuznetsov@telecom-paristech.fr

Matteo Monti

matteo.monti@epfl.ch

Dragos-Adrian Seredinschi

dragos-adrian.seredinschi@epfl.ch

1 École Polytechnique Fédérale de Lausanne (EPFL),

Lausanne, Switzerland

2 LTCI, Télécom Paris, Institut Polytechnique de Paris, Paris, France 